as the Fresno. Students found it very useful as a learning resource, demonstrating the application of EBM steps of asking, acquiring, appraising and applying evidence to a realistic clinical scenario. It is feasible to administer the ACE tool as a formative assessment in undergraduate medical education. It is a valuable teaching tool to demonstrate the application of the first four steps of EBM to a clinical scenario. Further research is needed to compare feasibility and students' performances in assessment tools and suggest a taxonomy of tools to guide EBM educators.

\section{EVIDENCE-BASED MEDICINE COURSE AS PART OF AN INTERNATIONAL MEDICAL EDUCATION CURRICULUM IN A RUSSIAN MEDICAL SCHOOL}

${ }^{1}$ Ksenia Ershova, ${ }^{2}$ Sergey Astrakov, ${ }^{1}$ Vladimir Zelman, ${ }^{2}$ Elena Neporada, ${ }^{1}$ Holly Muir, ${ }^{1}$ Philip Lumb. 'Department of Anesthesiology, Keck School of Medicine at the University of Southern California, Los Angeles, USA; '2Department of Anesthesiology and Intensive Care, Vladimir Zelman School of Medicine and Psychology at the Novosibirsk State University, Novosibirsk, Russian Federation

\subsection{6/bmjebm-2019-EBMLive.45}

Objectives Russian medical schools are currently incorporating evidence-based medicine (EBM) principles into their curricula. However, many medical schools still lack ancillary courses on research methods, epidemiology, biostatistics, etc., that result in reportedly poor quality of medical research in Russia. ${ }^{1} 2$ Many reasons for the difficulties have been identified, including linguistic barriers, lack of expertise, limited international collaboration, and inadequate allocation of resources. We hypothesized that the addition of an elective EBM curriculum to students in a Russian medical school would develop skills in searching, interpreting and evaluating medical literature, essential components of EBM.

As part of an international collaborative education program between two departments of anesthesiology, the EBM elective curriculum was created and introduced to all medical students (years 1-6). We then assessed the value of the course, students' attitude towards EBM, the extent to which they are engaged with evidence-based methodology, and the ability to incorporate EBM principles into future practice.

Method The collaborative education program was created between the Department of Anesthesiology, Keck School of Medicine at the University of Southern California (KSOM, USA) and the Department of Anesthesiology, School of Medicine and Psychology at the Novosibirsk State University (NSU, Russia). The EBM course included 18 seminars and journal clubs on biostatistics, clinical epidemiology, research methodology and other aspects of EBM. Classes were transmitted through video chat from KSOM to NSU, were voluntary and held in English. The course evaluation took place at the conclusion of the first year and consisted of two questionnaires. The first (Qs\#1) was for the course participants and a second (Qs\#2) was generally distributed among all students to learn about the potential course benefits and their understanding of EBM principles and importance. The questionnaires were distributed online as a GoogleForm (all questions were mandatory, one response per IP) and responses were anonymous.

Results Twenty-five students participated in the course. From 152 responses on Qs\#1, only 76\% agreed that clinical decision-making should be based on scientific evidence. A median frequency of reading medical literature was reported as once a week in their native language and once a month in English. On a scale from 1 to 10 , respondents ranked their understanding of scientific manuscripts as $6.3 \pm 1.8$, their confidence in assessing the quality of research as $5.5 \pm 2.1$. Respondents rated their interest in staying current with literature as $9.0 \pm 1.5$, and a potential benefit from learning critical thinking as $8.8 \pm 1.6$.

In a set of matched questions in Qs\#2, course participants reported that the course was valuable, helped build confidence in assessing research articles, and improved critical thinking. Participants vs. non-participants are more familiar with EBM $(8.2 \pm 2.2$ vs. $6.6 \pm 2.8, \mathrm{p}$-value $=0.002)$ and read medical literature more frequently (native language $p$-value $=0.039$, English p-value $=0.003$ ).

Conclusions Based on prior publications, ${ }^{1}{ }^{2}$ some Russian medical schools lack an EBM curriculum. By surveying a general medical student population, we found a discrepancy between the current and desired level of understanding of EBM indicating an unmet need. Currently, students feel insecure in applying EBM principles and have a deficiency in reading medical literature, especially in English. The EBM course, one component of an international education program, was established to create a scientific-centered learning environment, to provide expertise in EBM, and to help students overcome linguistic barrier. The participant evaluations showed that the course addressed positively the demonstrated needs of students and improved their reading and understanding of medical literature. We present evidence that reflects need for and benefits of including EBM in the medical curriculum. These findings indicate that international collaboration enhanced the medical students' educational experience by augmenting previously unavailable curricular content and should be continued.

\section{REFERENCES}

1. Telen, Marilyn J. 2014. "Teaching Evidence-Based Medicine in the Former Soviet Union: Lessons Learned." Transactions of the American Clinical and Climatological Association 125: 88-102; discussion 102-3. https://www.ncbi.nlm.nih.gov/ pubmed/25125721.

2. Vlassov, Vasiliy V. 2017. "Russian Medicine: Trying to Catch up on Scientific Evidence and Human Values." The Lancet 390 (10102): 1619-20. https://doi.org/ 10.1016/S0140- 6736(17)32382-6.

\section{TEACHING EVIDENCE BASED PRACTICE - IT'S TIME TO TACKLE THE 'APPLY' DOMAIN}

${ }^{1}$ Eve O'Toole, ${ }^{2}$ Niamh O'Rourke. 'National Cancer Control Programme, Health Service Executive, Dublin, Ireland; ${ }^{2}$ Clinical Effectiveness Unit, Department of Health, Dublin, Ireland

\subsection{6/bmjebm-2019-EBMLive.46}

Objectives In 2017 Evidence Based Practice Ireland (EBPI) was established to promote the use of evidence-based practice in the healthcare system in Ireland with the ultimate goal of improving patient outcomes. One of the challenges to the widespread adoption of the EBP paradigm is the lack of teaching of EBP to healthcare professionals. The teaching taking place rarely addresses the domain of how to apply evidence in practice. To address this deficit a three-day workshop in EBP was held in Ireland in March 2019 for practicing healthcare professionals with an emphasis on putting evidence into practice. The aims of the workshop were to develop and deliver a teaching module on 'evidence into practice'. To empower healthcare professionals to become evidence-based practitioners. To build capacity in teaching EBP and to create international links by inviting speakers form the Centre for Evidence Based Medicine (CEBM) in Oxford. 
Method The workshop was delivered through a blend of plenary and small group sessions modelled on the CEBM approach to teaching EBP. In all plenary's speakers used real life examples from their own experience with consistent emphasis on the impact to patients and the importance of patient values.

The programme for day one covered the Ask and Search domains. Day two explored appraisal of different study designs. Day three focused on putting evidence into practice with plenaries on

- From Evidence to recommendations

- Shared decision making

- Implementation planning

These topics were chosen to reflect the real-word challenges health care professionals face. Each small group was required to demonstrate the skills attained including, asking an answerable question in PICO format, finding the evidence, critical appraisal of the best evidence and how they would put the evidence into practice in the real-world setting

Results The small group presentations covered a wide range of topics which were based on a real clinical query of a participant. Each group discussed the quality of the evidence to answer their question, the potential benefit and harm, patient preferences and values and resources required before presenting their recommendations. They also described barriers and facilitators to the implementation of their recommendations.

In the evaluation form all participants rated their ability to practice EBP a 4-5 on a five-point scale compared with a range of $2-5$ in a previous workshop. When asked what aspects of the course were most useful, comments included;

'Practical aspects of bringing EBP to patients'

'Guidelines to aid implementation'

'Connection of theory to real world practice'

'Shared decision making'

'Apply information in practice'

Conclusions Our goal in EBPI is to promote the practice of EBP throughout the healthcare system in Ireland to improve patient outcomes. To be successful we need health care professionals to understand the principles of EBP. Much work internationally has gone into addressing the skills needed to ask focused questions, search the literature and appraise the literature for its quality and applicability. However, there is less agreement on how to teach integrating the best evidence with clinical expertise and patient values and applying it in practice. By developing this 'evidence into practice' module we are addressing the curriculum gap in how to teach the 'apply in practice' domain of EBP while building capacity and leadership in EBP in Ireland.

\section{EFFECT OF AN EBP LEARNING PROGRAM ON CONFIDENCE AND COMPETENCE IN EBP}

Michael Pianta, Edward Nguyen, Laura Downie. The University of Melbourne, Melbourne, Australia

\subsection{6/bmjebm-2019-EBMLive.47}

Objectives The aim of this study was to investigate the effect of an EBP learning program on learner confidence and competence in the use of EBP.
Method The Evidence-Based Practice Confidence (EPIC) scale (Salbach and Jaglal, J Eval Clin Pract, 2011) and the Assessing Competency in EBM (ACE) tool (Ilic et al, BMC Med Ed, 2014) were used to measure learner $(n=49)$ competence and confidence before and after a one-semester EBP learning program that incorporated the CrowdCARE platform.

Results The program resulted in a significant increase in both confidence $(+23.0 \%, 95 \%$ CI $[+18.2 \%,+27.8 \%])$ and competence $(+38.0 \%, 95 \%$ CI $[+33.3 \%,+42.8 \%])$ in the use of EBP. The largest increases in confidence were in critically appraising measurement properties, interpreting statistical tests, and integrating research evidence, clinical judgment and patient preferences; the largest increases in competence were in asking an answerable question and appraising the evidence. The smallest increases in confidence were in identifying gaps in knowledge and conducting a literature search; the smallest increase in competence was in applying the evidence.

Conclusions The learning program was effective at improving confidence and competence in EBP. The use of the EPIC scale and ACE tool allows identification of areas for improvement.

\section{PHARMACOLOGIC TREATMENT OF MAJOR DEPRESSION: MAKING RESEARCH EVIDENCE AVAILABLE WITH A PEER-REVIEWED ALGORITHM IN AN EASY TO USE COMPUTER PROGRAM}

${ }^{1,2}$ David Osser, ${ }^{1,3}$ Robert Patterson. ${ }^{1}$ Harvard Medical School, Boston, MA, USA; ${ }^{2}$ VA Boston HealthCare System, Brockton Division, Brockton, MA, USA; ${ }^{3}$ McLean Hospital, Belmont, MA, USA

\subsection{6/bmjebm-2019-EBMLive.48}

Objectives The Psychopharmacology Algorithm Project at the Harvard South Shore Psychiatry Department has been preparing evidence-derived algorithms for choosing medication for psychiatric disorders for 25 years. They provide clinicians with syntheses of the best evidence for efficacy, effectiveness, safety, and cost of pharmaceuticals in a format that is more prescriptive than guidelines. The aim is to present very specific and very context sensitive best practice. The algorithms start with recommendations for medication-naive patients. After that, suggestions for patients who had one failed trial are offered, and then we address deeper levels of treatmentresistance. Important comorbidity is considered. They are usable on cell phones. Practitioners can obtain an actionable recommendation in 1-2 minutes. We will demonstrate the major depression algorithm, most recently published in the February 2019 issue of Harvard Review of Psychiatry.

Method Small groups of clinicians create new algorithms or update older ones and then submit papers describing them for peer review in psychiatric journals. The review process involves reaching consensus with the expert reviewers who may not agree with all the interpretations of the evidence leading to the recommendations in the initial draft. This review process enhances the validity of the final version. After acceptance, the authors construct the on-line versions of the algorithms. Users see the entire flowchart and click on the node representing their patient's current clinical status - ranging from first use of medication to very treatment resistant, while considering the impact of various illness subtypes and comorbidities. The website (www.psychopharm.mobi) currently has 9 algorithms. All algorithms emphasize that they advise on choice of pharmacotherapy only, but non-medication approaches are valid, important, and may be preferred. 